\title{
Dyspareunia, CTCAE
}

National Cancer Institute

\section{Source}

National Cancer Institute. Dyspareunia, CT CAE. NCI Thesaurus. Code C143424.

A disorder characterized by painful or difficult coitus. 